\title{
Glutamate Mediates Acute Glucose Transport Inhibition in Hippocampal Neurons
}

\author{
Omar H. Porras, Anitsi Loaiza, and L. Felipe Barros \\ Centro de Estudios Científicos, Casilla 1469, Valdivia, Chile
}

\begin{abstract}
Although it is known that brain activity is fueled by glucose, the identity of the cell type that preferentially metabolizes the sugar remains elusive. To address this question, glucose uptake was studied simultaneously in cultured hippocampal neurons and neighboring astrocytes using a real-time assay based on confocal epifluorescence microscopy and fluorescent glucose analogs. Glutamate, although stimulating glucose transport in astrocytes, strongly inhibited glucose transport in neurons, producing in few seconds a 12 -fold increase in the ratio of astrocytic-to-neuronal uptake rate. Neuronal transport inhibition was reversible on removal of the neurotransmitter and displayed an $\mathrm{IC}_{50}$ of $5 \mu \mathrm{m}$, suggesting its occurrence at physiological glutamate concentrations. The phenomenon was abolished by CNQX and mimicked by AMPA, demonstrating a role for the cognate subset of ionotropic glutamate receptors. Transport inhibition required extracellular sodium and calcium and was mimicked by veratridine but not by membrane depolarization with high $\mathrm{K}^{+}$or by calcium overloading with ionomycin. Therefore, glutamate inhibits glucose transport via AMPA receptor-mediated sodium entry, whereas calcium entry plays a permissive role. This phenomenon suggests that glutamate redistributes glucose toward astrocytes and away from neurons and represents a novel molecular mechanism that may be important for functional imaging of the brain using positron emission tomography.
\end{abstract}

Key words: glucose; glutamate; neuron; membrane transport; 2-NBDG; 6-NBDG; AMPA

\section{Introduction}

Energy consumption in the mammalian brain is supplied by the oxidation of glucose. During the activation of discrete cortical areas, there is a correlative increase in local glucose uptake (Sokoloff, 1999) that is consistent with the high energetic cost of ion gradient restoration (Attwell and Laughlin, 2001). This link between neural activity and glucose uptake is exploited routinely for research and clinical purposes by means of positron emission tomography (PET) scanning, a noninvasive technique that follows the tissular uptake of a radioactive hexoses.

Because $85 \%$ of the energy expenditure of the brain occurs in neurons (Attwell and Laughlin, 2001) and glucose is almost the only fuel used by the organ, the natural assumption has been that neurons must feed on glucose. Such a conventional notion is consistent with the abundance of glucose transporters in these cells and the adequate electrical activity observed in cultured neurons grown in glucose-rich media. However, lactate is constitutively exported by astrocytes and can sustain firing in cultured neurons even more efficiently than glucose (Bouzier-Sore et al., 2003). Moreover, astrocytic lactate production is stimulated by the excitatory neurotransmitter glutamate (Pellerin and Magis-

Received May 14, 2004; revised Sept. 3, 2004; accepted Sept. 8, 2004.

This work was supported by Fondo Nacional de Desarrollo Científico y Tecnológico Grant 1020648. Centro de Estudios Científicos (CECS) is a Millennium Science Institute and is funded in part by Fundación Andes, the Tinker Foundation, and Empresas Compañia Manufacturera de Papeles y Cartones. We thank our colleagues at CECS for discussions and critical reading of this manuscript. We also thank two anonymous reviewers for helpful suggestions.

Correspondence should be addressed to Dr. Luis Felipe Barros, Centro de Estudios Científicos, Avenida Arturo Prat 154, Casilla 1469, Valdivia, Chile. E-mail: fbarros@cecs.cl.

DOI:10.1523/JNEUROSCI.1882-04.2004

Copyright $\odot 2004$ Society for Neuroscience $\quad$ 0270-6474/04/249669-05\$15.00/0 tretti, 1994), which, together with the activation of astrocytic glycogen degradation (Shulman et al., 2001), may explain the large increase in local extracellular lactate that follows cortical activation (Hu and Wilson, 1997). These observations support an alternative model of brain energy metabolism in which active neurons preferentially feed on lactate, which is produced by astrocytes from glucose and glycogen in response to neuronal glutamate (Magistretti et al., 1999).

Using real-time sugar transport assays, we were recently able to show that cultured astrocytes respond to glutamate by activating its glucose transporter GLUT1 on the order of seconds (Loaiza et al., 2003). This mechanism was proposed to make more glucose available to the astrocytic metabolism during and after neuronal activity. Exploiting the capability of these techniques to resolve single cells, we set out to investigate whether a similar mechanism was present in hippocampal neurons but found that, in these cells, glutamate exerts an effect opposite to that observed in astrocytes.

\section{Materials and Methods}

Materials. Fura Red AM, calcein AM, hexoses [2-[N-(7-nitrobenz-2-oxa1,3-diazol-4-yl)amino]-2-deoxyglucose (2-NBDG) and 6-[N-(7nitrobenz-2-oxa-1,3-diazol-4-yl)amino]-6-deoxyglucose (6-NBDG)], and pluronic acid were obtained from Molecular Probes (Eugene, OR). AMPA and CNQX were from Tocris-Cookson (Bristol, UK). Standard chemicals, tissue culture reagents, veratridine, ionomycin, and antiMAP1b (microtubule-associated protein $1 \mathrm{~b}$ ) were from Sigma (St. Louis, MO). Anti-GFAP was from Dako (High Wycombe, UK), and FITCconjugated and tetramethylrhodamine isothiocyanate-conjugated secondary antibodies were from Sigma.

Cell culture and immunodetection. Sprague Dawley rats were obtained 
from the Universidad Austral de Chile (Valdivia, Chile). Mixed cultures of neuronal and glial cells were prepared from 1- to 3-d-old neonatal as described by Loaiza et al. (2003). Briefly, cells were initially plated in MEM-10\% fetal bovine serum media and maintained at $37^{\circ} \mathrm{C}$ in a humid atmosphere with $5 \% \mathrm{CO}_{2}$ and $95 \%$ air. Two hours after plating, media was replaced by serum-free N1-MEM (MEM supplemented with 750 $\mathrm{mg} / \mathrm{ml}$ glucose, $100 \mu \mathrm{m}$ putrescine, $20 \mathrm{~nm}$ progesterone, $30 \mathrm{~nm}$ selenium dioxide, $100 \mu \mathrm{g}$ of transferrin, $5 \mu \mathrm{g} / \mathrm{ml}$ insulin, $1 \mathrm{~mm}$ sodium pyruvate, and $0.1 \%$ ovalbumin). Cells $\left(10^{5}\right)$ were plated on $1 \mathrm{mg} / \mathrm{ml}$ poly-L-lysinecoated coverslips $(25 \mathrm{~mm})$. One-half of the culture media (N1-MEM) was removed and replaced with new media $4 \mathrm{~d}$ after plating. Cells were used for experiments within $10 \mathrm{~d}$ of being cultured. Immunocytochemistry was performed using standard procedures described in detail previously (Barros et al., 1995). Paraformaldehyde-fixed cells were stained with affinity-purified antipeptide antibodies at $2 \mu \mathrm{g} / \mathrm{ml}$ (MAP1b) or 4 $\mu \mathrm{g} / \mathrm{ml}$ (GFAP).

Single-cell fluorescent hexose uptake by confocal microscopy. Cells were imaged using an inverted Zeiss (Oberkochen, Germany) LSM 5 PASCAL, laser-scanning confocal microscope with $40 \times$ (numerical aperture, 1.3) or $63 \times$ (numerical aperture, 1.4) objectives. Pinhole was set to produce optical sections thinner than $2 \mu \mathrm{m}$. Control experiments showed that under our experimental conditions, dye bleaching was negligible.

The uptake of the fluorescent hexoses 2-NBDG and 6-NBDG was assayed at room temperature $\left(23-26^{\circ} \mathrm{C}\right)$ as described by Loaiza et al. (2003). Before transport measurements, culture medium was removed and coverslips were washed with Krebs'-Ringer's-HEPES (KRH) buffer [in mM: $136 \mathrm{NaCl}, 20$ HEPES, $4.7 \mathrm{KCl}, 1.25 \mathrm{MgSO}_{4}$, and $1.25 \mathrm{CaCl}_{2}, \mathrm{pH}$ 7.4] supplemented with $3.3 \mathrm{~mm}$ glucose. For some experiments, cells were then loaded for 30 min with $5 \mu \mathrm{M}$ Fura Red AM. This allowed semiquantitative tracking of intracellular calcium. Five minutes before uptake, glucose in the medium was reduced to $0.5 \mathrm{~mm}$ to minimize competition with dye transport. Uptake was started by addition of 300 $\mu \mathrm{M}$ 2-NBDG or 6-NBDG, with the concentration chosen as the minimum capable of giving an adequate signal-to-noise ratio. Cultures were excited at $488 \mathrm{~nm}$. 2-NBDG and 6-NBDG were imaged at 505-550 nm emission, and Fura Red was imaged simultaneously at $>585 \mathrm{~nm}$ emission. Intracellular hexose concentration was calculated by comparing intracellular fluorescence with the signal outside of the cells. 6-NBDG is nonmetabolizable; thus, it directly reports transport. Because the effect of agonists and inhibitors was routinely assessed while the intracellular concentration of the sugar was $<25-30 \%$ of the extracellular, the tracer is said to be measured under near zero-trans conditions. In the case of 2-NBDG, which can be phosphorylated by hexokinase to give fluorescent 2-NBDG-6-phosphate, the concentration of free intracellular sugar is even lower, thus giving a better estimation of zero-trans uptake. Using computational analysis, we have shown previously that changes in phosphorylation are reflected on the uptake rate only after the intracellular concentration reaches $>50 \%$ of that in the extracellular (Quintanilla et al., 2000). In some experiments in which high concentrations of sugars were tested, $50 \mathrm{~mm} \mathrm{NaCl}$ in the buffer was replaced by $100 \mathrm{~mm} 4,6,-\mathrm{O}$ etilidene-D-glucose or $100 \mathrm{~mm}$ mannitol as a negative control.

Statistical analysis. Regression analyses were performed with SigmaPlot software (Jandel Scientific, Corte Madera, CA). Data are presented as means $\pm \operatorname{SEM}(n)$ throughout the paper.

\section{Results}

Identification of the cell type that preferentially captures and phosphorylates glucose in the brain has been hindered in vivo by the relatively low spatial resolution inherent to autoradiography and PET scanning and in vitro by the lack of single-cell resolution of standard isotope-based transport assays. With the aim of exploring the issue, we studied mixed hippocampal cultures in which neurons grew in intimate contact with astrocytes. The two cell types were identified morphologically and by the presence of specific cell markers (Fig. 1A). In agreement with the literature (Vannucci et al., 1997), both immunocytochemistry and immunoblotting showed that, under our culture conditions, astrocytes
A B
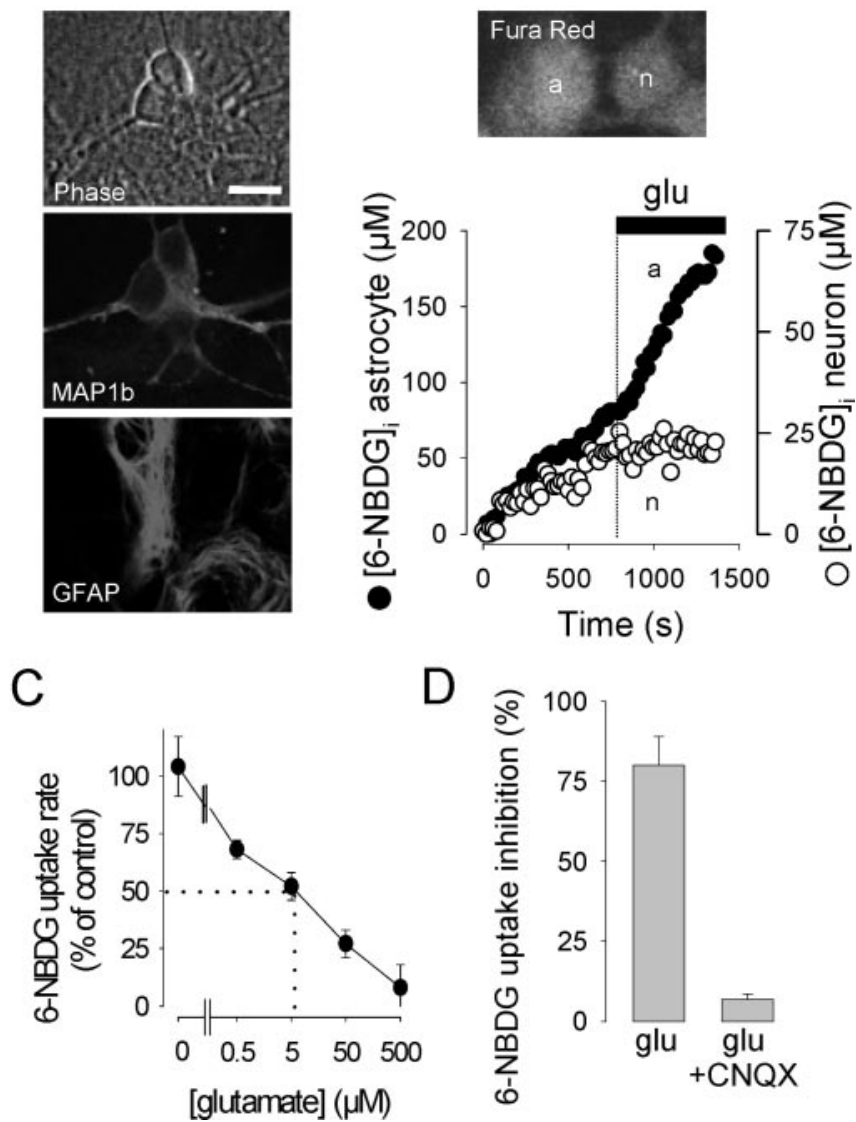

D

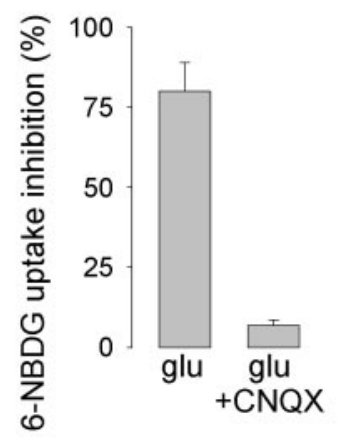

Figure 1. Glutamate inhibits glucose uptake by neurons. A, Imaged under phase contrast, living astrocytes were identified as flat polygonal sheets attached to the substrate, and neurons were defined as strongly birefringent bodies located on top of the astrocytic monolayer (top). These identification criteria were validated by costaining neurons for MAP1b (center) and astrocytes for GFAP (bottom). Scale bar, $10 \mu \mathrm{m}$. B, 6-NBDG uptake was measured by confocal microscopy in a single neuron (n) and a neighboring astrocyte (a). Scale bar, $10 \mu \mathrm{m}$. glu, Glutamate. At the time indicated (dashed line), $500 \mu \mathrm{m}$ glutamate was added to the culture. $C$, Hexose uptake rate as a function of glutamate concentration relative to the rate measured before glutamate addition ( $n>13$ neurons in 3-5 experiments for each concentration). Zero glutamate refers to mock addition of buffer. $D$, Inhibition of hexose uptake by $500 \mu \mathrm{m}$ glutamate in the absence and presence of $20 \mu \mathrm{M}$ CNQX.

expressed GLUT1, whereas neurons expressed GLUT3 (data not shown).

Glucose transport was measured using confocal microscopy and the fluorescent tracers 2-NBDG and 6-NBDG, which are substrates of GLUT carriers in a variety of cell types, including astrocytes (Loaiza et al., 2003) and neurons (Aller et al., 1997). As will be shown below, the modulation of the uptake rate triggered by agonists and inhibitors were similar for both dyes, indicating that the results are insensitive to the particular structure of the sugar tracer used. In preliminary experiments, the impermeant GLUT substrate 4,6,-O-etilidene-D-glucose (Carruthers, 1990) was found to inhibit the uptake of 6-NBDG in neurons and astrocytes by $84 \pm 5 \%[n=11$ cells, 3 experiments (exps.)] and $80 \pm 8 \%$ ( $n=7$ cells, 3 exps.), providing independent confirmation of the permeation route. In 29 experiments, the basal rate of 2-NBDG uptake was significantly higher in astrocytes than in neurons, with rates of $36 \pm 2.2 \mathrm{~nm} / \mathrm{sec}(n=82$ cells $)$ and $13 \pm 0.6$ $\mathrm{nm} / \mathrm{sec}(n=129$ cells), respectively. There was, however, considerable overlap between the two populations, with $>16 \%$ of the 
neurons displaying rates higher than the astrocytic average. For 6 -NBDG, the difference was not so marked with basal rates in astrocytes and neurons of $56 \pm 8 \mathrm{~nm} / \mathrm{sec}(n=14$ cells, 7 exps.) and $44 \pm 4 \mathrm{~nm} / \mathrm{sec}$, respectively. Because both $2-\mathrm{NBDG}$ and 6-NBDG uptakes were measured under near zero-trans conditions and thus represent transport and not metabolism (see Materials and Methods), we do not think the difference between the two cell types in the handling of the dyes is related to the capability of 2-NBDG to be phosphorylated by hexokinase. More likely, the divergence is related to the fact that astrocytes and neurons express different isoforms of the glucose transporter with different catalytic properties. Neuronal GLUT3 presents a much higher turnover number than astrocytic GLUT1 (Maher et al., 1996), so it is possible that the isoforms also differ to some extent in the way they bind-translocate sugars.

Glutamate triggered opposite responses in astrocytes and neurons $($ Fig. $1 B)$. Astrocytes reacted to glutamate $(500 \mu \mathrm{M})$ with a $151 \pm 21 \%$ stimulation of $6-\mathrm{NBDG}$ uptake $(n=14$ cells, 7 exps.), similar to the $178 \%$ reported previously (Loaiza et al., 2003). In stark contrast, the same concentration of glutamate inhibited neuronal uptake by $80 \pm 9 \%(n=31$ cells, 8 exps. $)$. This means that, in the presence of the neurotransmitter, the average ratio between astrocytic and neuronal hexose transport increased 12fold, from 1.27 to 15.8 . The inhibition of sugar uptake by glutamate was detected in $80-100 \%$ of the neurons present in a given field and was dose dependent, with an $\mathrm{IC}_{50}$ of $5 \mu \mathrm{M}$ (Fig. $1 C$ ). Maximum inhibition required concentrations higher than 100 $\mu \mathrm{M}$, well over the affinities of NMDA and metabotropic glutamate receptors but inside the dynamic range of ionotropic nonNMDA receptors. Consistently, 6-NBDG uptake inhibition was abolished by the non-NMDA receptor antagonist CNQX (Fig. $1 D)$. Similar results were obtained with 2-NBDG, whose basal rate of uptake by neurons was $90 \%$ inhibited by glutamate from $21 \pm 2$ to $2 \pm 1 \mathrm{~nm} / \mathrm{sec}(n=14$ cells, 3 exps.). In the presence of CNQX, glutamate failed to affect 2-NBDG uptake, with an inhibition of $-7 \pm 35 \%$ ( $n=14$ cells, 3 exps.). In the presence of 2.5 $\mathrm{mm}$ lactate, an alternative carbon source, glutamate appeared to be even more effective, with an inhibition of neuronal 6-NBDG transport of $96 \pm 2 \%$ ( $n=13$ cells, 3 exps.). In the presence of the glutamate transporter blocker DL-threo- $\beta$-benzyloxyaspartic acid $(200 \mu \mathrm{M})$, glutamate still inhibited hexose transport by $60 \pm$ $6 \%(n=16$ neurons, 5 exps.), ruling out a major role for these carriers, which are responsible for the activation of glycolysis in astrocytes (Voutsinos-Porche et al., 2003).

The effect of glutamate on hexose transport was mimicked by AMPA, demonstrating a role for the cognate subset of the nonNMDA receptor family (Fig. $2 A$ ). As expected, the effect of AMPA was inhibited pharmacologically by CNQX and functionally by the replacement of extracellular sodium with the impermeant cation $\mathrm{N}$-methyl-D-glucamine (Fig. $2 \mathrm{~B}$ ). Control experiments using the inert dye calcein as a volume reporter (Barros, $1999)$ showed that neither glutamate $(500 \mu \mathrm{M})$ nor AMPA (20 $\mu \mathrm{M})$ caused any detectable neuronal swelling under our experimental conditions (data not shown). Astrocytes did not respond to AMPA $(20 \mu \mathrm{M})$, with $2-\mathrm{NBDG}$ rates of $30 \pm 5$ and $28 \pm 5$ $\mathrm{nm} / \mathrm{sec}$ before and after treatment, respectively.

The AMPA receptor in hippocampal neurons behaves as a monovalent-specific cation channel (Dingledine et al., 1999) whose activation leads to sodium entry and plasma membrane depolarization. The latter triggers calcium entry via voltagedependent calcium channels, which in our experiments was detected as a $43 \pm 8 \%$ maximum fall in Fura Red fluorescence $(n=$ 24 neurons, 7 exps.). Experiments were subsequently performed
A

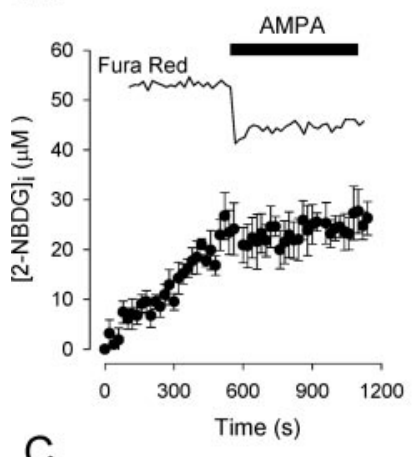

B
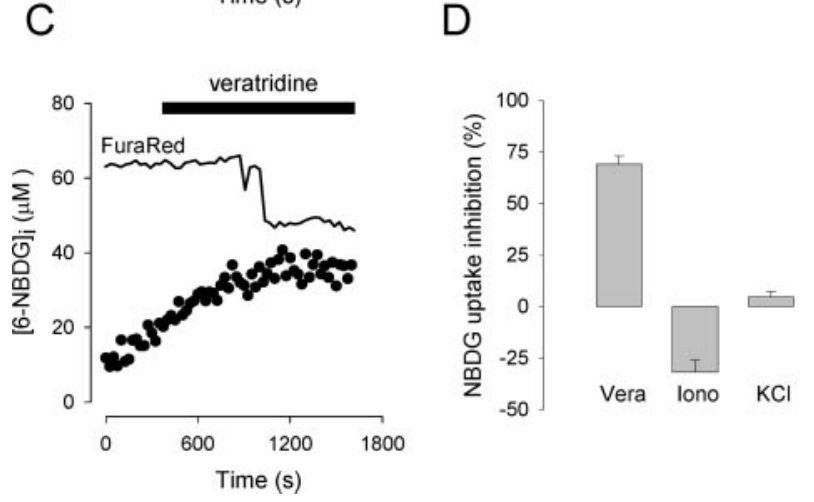

Figure 2. Sodium entry via AMPA receptors mediate neuronal glucose uptake inhibition. $A$, Hexose uptake by neurons was measured before and after the addition of $20 \mu \mathrm{M}$ AMPA. The top trace corresponds to simultaneous nonquantitative assessment of cytosolic calcium with the reciprocal dye Fura $\operatorname{Red}(n=6$ cells). $B$, Inhibition of hexose uptake elicited by AMPA alone ( $n=$ 24 cells, 7 exps.) in the presence of $20 \mu \mathrm{m}$ CNQX ( $n=9$ cells, 2 exps.) in a buffer in which $\mathrm{Na}^{+}$ was equimolarly replaced by $N$-methyl-D-glucamine ( $n=15$ cells, 3 exps.) and in the presence of $10 \mathrm{~mm} \mathrm{EGTA} \mathrm{(} n=22$ cells, 4 exps.). C, Hexose uptake by a neuron was measured before and after the addition of $75 \mu \mathrm{m}$ veratridine. The top trace represents the corresponding Fura Red signal.D, Inhibition of hexose uptake by veratridine (Vera) (6-NBDG; $n=18$ cells, 4 exps.), $2 \mu \mathrm{m}$ ionomycin (lono) (2-NBDG; $n=17$ cells, 3 exps.), and $40 \mathrm{~mm} \mathrm{KCI} \mathrm{(2-NBDG;} n=28$ cells, 6 exps.).

to understand how these diverse downstream events impinge on glucose transport. Sodium entry was mimicked with the alkaloid veratridine, which keeps sodium channels in a modified open state, causing sodium overload (Hille, 2001). When applied to hippocampal neurons, veratridine effectively inhibited glucose transport similar to that seen with glutamate and AMPA (Fig. $2 C, D)$. The response was typically delayed with respect to the application of the toxin (for instance, $\sim 10 \mathrm{~min}$ ) (Fig. $2 \mathrm{C}$ ), which can be explained by the mechanism of action of veratridine, which prevents the closing of voltage-dependent sodium channels only when they open spontaneously (Hille, 2001). In healthy, nonstimulated neurons, the plasma membrane is polarized, and spontaneous sodium channel opening is a rare event. When the toxin binds to an open channel, it depolarizes the plasma membrane slightly, increasing the probability for other channels to open and thus bind the toxin. The result is a highly regenerative process that may be delayed. This explanation is substantiated by the observation that the calcium rise, as a result of secondary opening of voltage-sensitive calcium channels, was also delayed (Fig. 2C). Membrane depolarization by itself does not seem to play a direct role because a high concentration of potassium chloride failed to modify hexose uptake (Fig. $2 D$ ). Incidentally, with its $51 \pm 8 \%$ maximum fall in Fura Red fluorescence $(n=28$ neurons, 6 exps.), the potassium experiment also demonstrates that calcium is not sufficient to inhibit hexose uptake. Direct demonstration of the point was afforded by the calcium iono- 
A

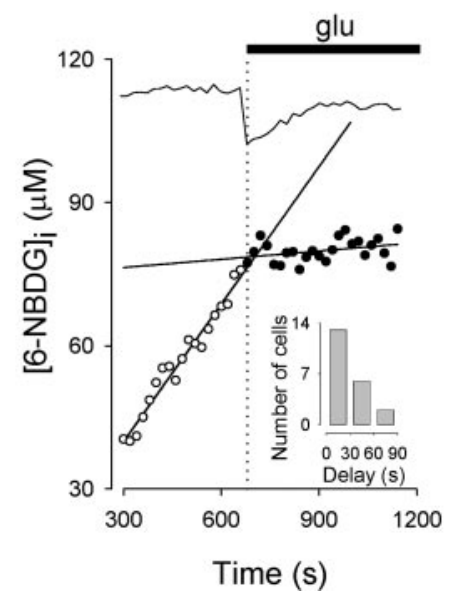

B

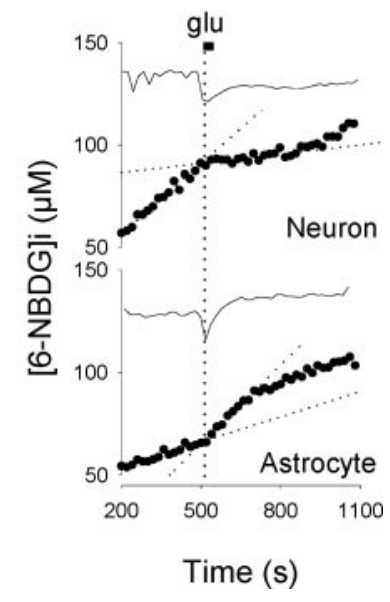

Figure 3. Glutamate (glu) inhibition of neuronal glucose uptake is fast and reversible. $A$, Linear functions were fitted to neuronal hexose uptake data before (open circles) and after (filled circles) the addition of $500 \mu \mathrm{m}$ glutamate. The intercept between the two curves was compared with the onset of the calcium deflection (top trace) to obtain the delay of transport inhibition. For this particular neuron, the delay was $24 \mathrm{sec}$. Inset, Frequency distribution for 21 neurons (7 exps.) exposed to glutamate. $B$, A neuron and a neighboring astrocyte were exposed to $500 \mu \mathrm{m}$ glutamate for $25 \mathrm{sec}$. Note that, after transient changes, transport rates returned to basal values in both cells. Data are representative of at least four independent experiments.

phore ionomycin, which produced a $52 \pm 12 \%$ maximum decrease in Fura Red signal ( $n=17$ neurons, 3 exps.), but again failed to inhibit glucose transport (Fig. 2D). Nevertheless, calcium entry was found to be necessary because removal of extracellular calcium with EGTA abolished both the effect of AMPA (Fig. $2 B$ ) and the calcium increase $(-1 \pm 12 \%$ decrease in Fura Red signal; $n=22$ neurons, 4 exps.). Together, these results show that sodium and calcium entry are the only signals required downstream of the AMPA receptor in its modulation of glucose transport.

In astrocytes, the change in the rate of glucose transport can be detected within seconds of the calcium transient elicited by glutamate (Loaiza et al., 2003). Our present results indicate that the same is valid for neurons, with the majority of the cells presenting delays shorter than $30 \mathrm{sec}$ (Fig. 3A). These responses to glutamate in brain cells represent the fastest modulations of glucose transport yet reported. More importantly, when the agonist was removed, both neurons and astrocytes recovered their basal uptake rates (Fig. $3 B$ ), demonstrating that the effects are reversible and therefore of possible physiological interest.

\section{Discussion}

Neurons consume $>10$ times more energy than astrocytes (Attwell and Laughlin, 2001), and all of that energy is supplied by blood-borne glucose. Paradoxically, the data reported here show that, after activation, neurons actually decrease their glucose uptake in favor of astrocytes. Notwithstanding extrapolation caveats that are discussed below, the question arises as to the identity of the fuel that sustains neurons during and after firing. Pellerin and Magistretti proposed that neuronal firing may be supported preferentially by lactate (Pellerin and Magistretti, 1994; Magistretti et al., 1999), which is released by astrocytes as a result of glutamate activation of glycolysis (Pellerin and Magistretti, 1994), likely aided by accelerated glycogenolysis (Shulman et al., 2001) and stimulated glucose transport (Loaiza et al., 2003). This astrocyteto-neuron lactate shuttle has received recent in vivo support from experiments in knock-out mice (Voutsinos-Porche et al., 2003).
However, the hypothesis remains controversial, and its critics have pointed out that glucose metabolism, by competing for $\mathrm{NAD}^{+}$, should obstruct lactate usage by neurons (Chih et al., 2001). The argument follows that lactate may only be used by neurons if their glycolytic flux is small, which is not to be expected because these cells are richly endowed with glucose transporters (Vannucci et al., 1997).

Our data show that early during neuronal activation, a specific mechanism triggered by glutamate and dependent on the influx of sodium via AMPA receptors actively prevents glucose from entering neurons. Considering the competition described between glucose and lactate for $\mathrm{NAD}^{+}$, the rapid inhibition of glucose uptake in neurons, by providing a $\mathrm{NAD}^{+}$surge, should help to create a thermodynamic sink for lactate. Seconds later, when the lactate wave from astrocytes reaches the neuron, $\mathrm{NAD}^{+}$depletion may further inhibit glycolytic flux by functional blockage of glyceraldehyde-3-phosphate dehydrogenase via a mechanism called the redox switch (Cruz et al., 2001). Of note, our experiments showed that lactate does not interfere with the effect of glutamate on glucose transport, so both pathways of inhibition may work in synergy. The preferential use of lactate is supported by a recent in vitro nuclear magnetic resonance study in which cultured neurons preferred lactate over glucose (Bouzier-Sore et al., 2003) and previous in vivo measurements in the hippocampus showing that stimulation of glutamatergic afferents cause a fast decrease in extracellular lactate, presumably attributable to neuronal uptake (Hu and Wilson, 1997). The reciprocal effects of glutamate on glucose transport by astrocytes and neurons help to explain why sugar uptake in vivo correlates better with the expression of GLUT1 (found in astrocytes) than GLUT3 (found in neurons) (Duelli et al., 2001). Extrapolating to the intact brain, we endorse the view that fluorodeoxyglucose-PET imaging, which is based on local accumulation of a glucose derivative, also on the order of seconds (Sokoloff, 1999), reports mainly an astrocytic phenomenon, although directly controlled by neuronal firing. An analogous conclusion was reached recently regarding the brain microcirculation in which astrocytes trigger local vasodilation when activated by glutamate (Zonta et al., 2003).

Although $0.5 \mathrm{~mm}$ glutamate were required to obtain full inhibition, micromolar concentrations did have a significant effect, implying that the mechanism may be triggered outside the synapse. As a reference, glutamate reaches micromolar levels in the synaptic cleft during firing (Clements et al., 1992), with a spillover of 160-190 $\mu \mathrm{M}$ estimated in extrasynaptic regions (Dzubay and Jahr, 1999). Glucose transport in other tissues, such as muscle and fat, behaves as a local phenomenon whose characteristics can be studied adequately at room temperature in primary cultured cells and even in transformed cell lines (Baldwin, 1993). For this reason, we expect the results from the present study to be relevant to the intact tissue. However, cultured cells do not necessarily retain the properties of the original tissue, and it is possible that brain neurons in situ behave differently. Another caveat relates to the fact that transport measurements in neurons were made in cell bodies and therefore should only be considered to be representative of the transport properties of somas and possibly of proximal dendrites.

To the best of our knowledge, the effect of glutamate on neurons is the first description of a physiological inhibition of glucose transport. It is remarkable that a single agonist may cause such divergent responses in astrocytes and neurons. The two cell types differ in many aspects, particularly at both ends of the signaling pathway, displaying different glutamate receptors-transporters and also different GLUT isoforms. However, the response in neu- 
rons required sodium influx, and although it is not known at this stage whether sodium is involved in astrocytic GLUT1 stimulation, sodium influx does mediate the activation of astrocytic glycolysis by glutamate (Pellerin and Magistretti, 1997; VoutsinosPorche et al., 2003). Sodium appears, therefore, as a common signal downstream of glutamate in its diversion of glucose metabolism from neurons to astrocytes. In other cell types, calcium can also play a permissive role on acute glucose transport modulation (Draznin et al., 1987; Quintanilla et al., 2000). In epithelial cells, GLUT1 modulation by metabolic stress is associated with the activation of the AMP-activated protein kinase (Abbud et al., 2000; Barnes et al., 2002), an enzyme that may be sensitive to the increased ATP expenditure caused by glutamate-induced sodium entry in neurons. However, transport modulation in other cell types develops in minutes to hours and leads to transporter stimulation, so it does not seem likely that the effect of glutamate on neurons, which develops in seconds and results in transporter inhibition, will be mediated by a similar mechanism.

In summary, data from a variety of sources indicate that firing neurons feed preferentially on lactate, whose production by neighboring astrocytes is increased by the combined effects of accelerated glycogenolysis, stimulated glucose transport, and accelerated glycolysis. The present data supports this model by providing a molecular mechanism that diverts glucose from neurons to astrocytes and that allows neurons preferentially to metabolize lactate over glucose.

\section{References}

Abbud W, Habinowski S, Zhang JZ, Kendrew J, Elkairi FS, Kemp BE, Witters LA, Ismail-Beigi F (2000) Stimulation of AMP-activated protein kinase (AMPK) is associated with enhancement of Glut1-mediated glucose transport. Arch Biochem Biophys 380:347-352.

Aller CB, Ehmann S, Gilman-Sachs A, Snyder AK (1997) Flow cytometric analysis of glucose transport by rat brain cells. Cytometry 27:262-268.

Attwell D, Laughlin SB (2001) An energy budget for signaling in the grey matter of the brain. J Cereb Blood Flow Metab 21:1133-1145.

Baldwin SA (1993) Mammalian passive glucose transporters: members of an ubiquitous family of active and passive transport proteins. Biochim Biophys Acta 1154:17-49.

Barnes K, Ingram JC, Porras OH, Barros LF, Hudson ER, Fryer LG, Foufelle F, Carling D, Hardie DG, Baldwin SA (2002) Activation of GLUT1 by metabolic and osmotic stress: potential involvement of AMP-activated protein kinase (AMPK). J Cell Sci 115:2433-2442.

Barros LF (1999) Measurement of sugar transport in single living cells. Pflügers Arch 437:763-770.

Barros LF, Yudilevich DL, Jarvis SM, Beaumont N, Young JD, Baldwin SA (1995) Immunolocalisation of nucleoside transporters in human placental trophoblast and endothelial cells: evidence for multiple transporter isoforms. Pflügers Arch 429:394-399.

Bouzier-Sore AK, Voisin P, Canioni P, Magistretti PJ, Pellerin L (2003) Lactate is a preferential oxidative energy substrate over glucose for neurons in culture. J Cereb Blood Flow Metab 23:1298-1306.

Carruthers A (1990) Facilitated diffusion of glucose. Physiol Rev 70:1135-1176.
Chih CP, Lipton P, Roberts Jr EL (2001) Do active cerebral neurons really use lactate rather than glucose? Trends Neurosci 24:573-578.

Clements JD, Lester RA, Tong G, Jahr CE, Westbrook GL (1992) The time course of glutamate in the synaptic cleft. Science 258:1498-1501.

Cruz F, Villalba M, Garcia-Espinosa MA, Ballesteros P, Bogonez E, Satrustegui J, Cerdan S (2001) Intracellular compartmentation of pyruvate in primary cultures of cortical neurons as detected by (13)C NMR spectroscopy with multiple (13)C labels. J Neurosci Res 66:771-781.

Dingledine R, Borges K, Bowie D, Traynelis SF (1999) The glutamate receptor ion channels. Pharmacol Rev 51:7-61.

Draznin B, Sussman K, Kao M, Lewis D, Sherman N (1987) The existence of an optimal range of cytosolic free calcium for insulin-stimulated glucose transport in rat adipocytes. J Biol Chem 262:14385-14388.

Duelli R, Maurer MH, Staudt R, Sokoloff L, Kuschinsky W (2001) Correlation between local glucose transporter densities and local 3-Omethylglucose transport in rat brain. Neurosci Lett 310:101-104.

Dzubay JA, Jahr CE (1999) The concentration of synaptically released glutamate outside of the climbing fiber-Purkinje cell synaptic cleft. J Neurosci 19:5265-5274.

Hille B (2001) Modification of gating in voltage-sensitive channels. In: Ion channels of excitable membranes, Chap 20. Sunderland, MA: Sinauer Associates.

Hu Y, Wilson GS (1997) A temporary local energy pool coupled to neuronal activity: fluctuations of extracellular lactate levels in rat brain monitored with rapid-response enzyme-based sensor. J Neurochem 69:1484-1490.

Loaiza A, Porras OH, Barros LF (2003) Glutamate triggers rapid glucose transport stimulation in astrocytes as evidenced by real-time confocal microscopy. J Neurosci 23:7337-7342.

Magistretti PJ, Pellerin L, Rothman DL, Shulman RG (1999) Energy on demand. Science 283:496-497.

Maher F, Davies-Hill TM, Simpson IA (1996) Substrate specificity and kinetic parameters of GLUT3 in rat cerebellar granule neurons. Biochem J 315:827-831.

Pellerin L, Magistretti PJ (1994) Glutamate uptake into astrocytes stimulates aerobic glycolysis: a mechanism coupling neuronal activity to glucose utilization. Proc Natl Acad Sci USA 91:10625-10629.

Pellerin L, Magistretti PJ (1997) Glutamate uptake stimulates $\mathrm{Na}^{+}, \mathrm{K}^{+}$ATPase activity in astrocytes via activation of a distinct subunit highly sensitive to ouabain. J Neurochem 69:2132-2137.

Quintanilla RA, Porras OH, Castro J, Barros LF (2000) Cytosolic [Ca(2+)] modulates basal GLUT1 activity and plays a permissive role in its activation by metabolic stress and insulin in rat epithelial cells. Cell Calcium 28:97-106.

Shulman RG, Hyder F, Rothman DL (2001) Cerebral energetics and the glycogen shunt: neurochemical basis of functional imaging. Proc Natl Acad Sci USA 98:6417-6422.

Sokoloff L (1999) Energetics of functional activation in neural tissues. Neurochem Res 24:321-329.

Vannucci SJ, Maher F, Simpson IA (1997) Glucose transporter proteins in brain: delivery of glucose to neurons and glia. Glia 21:2-21.

Voutsinos-Porche B, Bonvento G, Tanaka K, Steiner P, Welker E, Chatton JY, Magistretti PJ, Pellerin L (2003) Glial glutamate transporters mediate a functional metabolic crosstalk between neurons and astrocytes in the mouse developing cortex. Neuron 37:275-286.

Zonta M, Angulo MC, Gobbo S, Rosengarten B, Hossmann KA, Pozzan T, Carmignoto G (2003) Neuron-to-astrocyte signaling is central to the dynamic control of brain microcirculation. Nat Neurosci 6:43-50. 